\title{
HOMOMORPHISMS OF LATTICES OF CONTINUOUS FUNCTIONS
}

\author{
R. MENA AND B. ROTH
}

\begin{abstract}
We prove that for $Y$ a compact Hausdorff space, every lattice homomorphism from $C(Y)$ to $C(X)$ which takes each constant function on $Y$ to the same function on $X$ is linear.
\end{abstract}

Let $C(X)$ be the space of real-valued continuous functions on the topological space $X$. Pointwise operations make $C(X)$ into a ring, an algebra, and a lattice. If $\phi: X \rightarrow Y$ is continuous, then $\phi$ induces a map $C(\phi): C(Y) \rightarrow$ $C(X)$ defined for all $f \in C(Y)$ by

$$
C(\phi)(f)=f \circ \phi .
$$

Clearly $C(\phi)$ is a ring, algebra, and lattice homomorphism which fixes each constant function, i.e., $C(\phi)\left(r_{Y}\right)=r_{X}$ for all $r \in \mathbf{R}$. It is well known that for $Y$ a compact Hausdorff space, every ring homomorphism from $C(Y)$ to $C(X)$ taking $1_{Y}$ to $1_{X}$ arises in this way.

THEOREM. Suppose $Y$ is a compact Hausdorff space and $\Phi: C(Y) \rightarrow C(X)$ is a lattice homomorphism such that $\Phi\left(r_{Y}\right)=r_{X}$ for all $r \in \mathbf{R}$. Then there exists a continuous map $\phi: X \rightarrow Y$ such that $\Phi=C(\phi)$.

We regard a prime ideal $P$ as a nonempty, proper lattice ideal with $f \in P$ or $g \in P$ whenever $f \wedge g \in P$. Following Kaplansky [2], we say that a prime ideal $P$ in $C(X)$ is associated with a point $x \in X$ if $g \in P$ whenever $f \in P$ and $g(x)<f(x)$. For $X$ compact, every prime ideal in $C(X)$ is associated with some point of $X$ and this point is unique if $X$ is also Hausdorff [2, Lemma 3]. Moreover, it is easy to see that if $P \subset Q$ where $P, Q$ are prime ideals in $C(X), X$ a compact Hausdorff space, then $P$ and $Q$ are associated with the same point.

For $x \in X$, let $\delta_{x}: C(X) \rightarrow \mathbf{R}$ be the point evaluation map defined for $f \in C(X)$ by

$$
\delta_{x}(f)=f(x) .
$$

Lemma. Suppose $X$ is a compact Hausdorff space and $\Phi: C(X) \rightarrow \mathbf{R}$. Then $\Phi$ is a lattice homomorphism satisfying $\Phi\left(r_{X}\right)=r$ for all $r \in \mathbf{R}$ if and only if $\Phi=\delta_{x}$ for some $x \in X$.

Proof. For $a \in \mathbf{R}$, let $P_{a}=\{r \in \mathbf{R}: r \leqslant a\}$. Since $\left\{P_{a}: a \in \mathbf{R}\right\}$ is a chain

Received by the editors July 21, 1977 and, in revised form, December 15, 1977. AMS (MOS) subject classifications (1970). Primary 06A70, 46E05; Secondary 46A40. 
of prime ideals in $\mathbf{R},\left\{\Phi^{-1}\left(P_{a}\right): a \in \mathbf{R}\right\}$ is a chain of prime ideals in $C(X)$ and hence each $\Phi^{-1}\left(P_{a}\right)$ is associated with the same point $x \in X$. We show that $\Phi(f)=f(x)$ for all $f \in C(X)$, i.e., $\Phi=\delta_{x}$. For $r \in \mathbf{R}, r<f(x)$ implies $r_{X} \in \Phi^{-1}\left(P_{\Phi(f)}\right)$ since $\Phi^{-1}\left(P_{\Phi(f)}\right)$ is associated with $x$ and thus $r=\Phi\left(r_{X}\right) \leqslant$ $\Phi(f)$. Hence $f(x) \leqslant \Phi(f)$. And for $r \in \mathbf{R}, f(x)<r$ implies $f \in \Phi^{-1}\left(P_{r}\right)$ so $\Phi(f) \leqslant r$. Therefore $\Phi(f)=f(x)$.

Proof of Theorem. For each $x \in X, \delta_{x} \circ \Phi: C(Y) \rightarrow \mathbf{R}$ is a lattice homomorphism fixing the constants and thus there exists a unique $y \in Y$ with $\delta_{x} \circ \Phi=\delta_{y}$. Letting $\phi(x)=y$, we have for all $f \in C(Y)$ and all $x \in X$

$$
\Phi(f)(x)=\left(\delta_{x} \circ \Phi\right)(f)=\delta_{y}(f)=(f \circ \phi)(x)
$$

and therefore $\Phi=C(\phi)$. The continuity of $\phi: X \rightarrow Y$ follows immediately since the zero sets $f^{-1}(0), f \in C(Y)$, form a base for the closed sets of $Y$. Pertinent examples appear in [1].

\section{REFERENCES}

1. K. Geba and Z. Semadeni, On linear isotonical embedding of $C\left(\Omega_{1}\right)$ into $C\left(\Omega_{2}\right)$, Studia Math. 19 (1960), 303-320.

2. I. Kaplansky, Lattices of continuous functions, Bull. Amer. Math. Soc. 53 (1947), 617-623.

3. __ Lattices of continuous functions. II, Amer. J. Math. 70 (1948), 626-634.

DEPARTMENT OF MATHEMATICS, UNIVERSITY OF WYOMING, LARAMIE, Wyoming 82071 